\section{Neonatal Candidemia Exacerbated the Progression of Retinopathy of Prematurity in an Extremely-Low-Birth- Weight Infant with Persistent Tunica Vasculosa Lentis}

\author{
Ching-ju Hsieh, MD \\ Keh-chung Lin, ScD, OTR \\ Jung-sen Liu, MD
}

\begin{abstract}
Eyes with threshold retinopathy of prematurity (ROP) often show dilation of the existing tunica vasculosa lentis (TVL), which is a prognostic factor in ROP screening and treatment, but can be frequently misdiagnosed as true iris neovascularization (NV). Besides, candidemia in preterm infants can aggravate the severity of ROP, possibly to the threshold stage requiring laser treatment. A case of prolonged candidemia without ophthalmic infection in an extremelylow-birth-weight (ELBW) (birth weight: $800 \mathrm{~g}$ ), preterm (gestation: 26 weeks) infant with persistent TVL and severe ROP are reported. The threshold ROP was successfully treated with argon laser therapy. Candidemia without eye infection in this case was associated with early use of a systemic antifungal drug. To our knowledge, no similar cases have been reported in the literature.
\end{abstract}

\section{INTRODUCTION}

Extremely-low-birth-weight (ELBW) and extreme prematurity are significant risk factors for candidemia ${ }^{1,2}$ and retinopathy of prematurity (ROP), ${ }^{3}$ in which retinal ischemia induces increased levels of vascular endothelial growth factor (VEGF) leading to retinal neovascularization (NV). ${ }^{4,5}$ Studies have shown that candidemia in preterm infants can aggravate the severity of ROP, possibly to the threshold stage requiring laser treatment. ${ }^{1,6-8}$ Embryologically, the hyaloid vascular system (HVS) provides the blood supply to the posterior portion of the tunica vasculosa lentis (TVL), a transiently existing network of capillaries that functions to nourish the developing lens. ${ }^{9}$ The TVL may persist after the HVS involutes and its regression is often particularly delayed in patients with ROP. ${ }^{1}$ Persistent TVL can be misdiagnosed as true iris NV in preterm infants with ROP. ${ }^{10}$ We reported a preterm infant with prolonged candidemia, persistent TVL, and threshold ROP, who was successfully treated with argon laser and with a systemic antifungal drug. To our knowledge, no similar cases have been reported in the open literature.

\section{CASE REPORT}

A boy was born at 26 weeks gestation, weighing 800 g. The pregnancy was complicated by premature labor because of maternal vaginal incontinence. Perinatal findings included extensive bruising during his entire body, immature skin, patent ductus arteriosus, and severe respiratory distress syndrome requiring immediate intubation and ventilatory support. The infant subsequently developed several complications, including necrotizing entercolitis, intraventricular hemor-

From the Department of Ophthalmology (C-JH), Taipei City Hospital, Heping Branch and Institute of Biophotonics, National Yang-Ming University, Taipei, Taiwan; the Division of Occupational Therapy (K-CL), Department of Physical Medicine and Rehabilitation, National Taiwan University Hospital and School of Occupational Therapy, College of Medicine, National Taiwan University, Taipei, Taiwan; and the Departments of Surgery and Medical Education (J-SL), Cathay General Hospital and School of Medicine, Fu-Jen Catholic University, Taipei, Taiwan.

Accepted for publication November 13, 2008. Posted online February 15, 2010.

The authors have no financial or proprietary interest in the materials presented herein.

Address correspondence to Jung-sen Liu, MD, 280 Section 4, Jen-Ai Road, Taipei, Taiwan, and Keh-chung Lin, ScD, OTR, 17, F4, Xu Zhou Road, Taipei, Taiwan. doi: $10.3928 / 15428877-20100215-12$ 


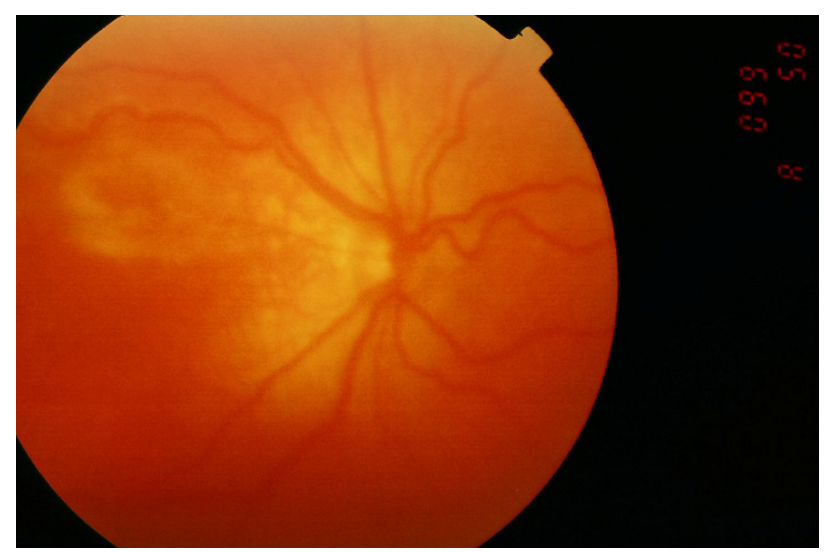

Figure 1. Plus disease in the right eye shows tortuous retinal arterioles and engorged veins close to the optic disc and posterior pole.

rhage, bilateral inguinal hernia, and several episodes of sepsis. Blood culture was positive for oxacillin-resistance staphylococcus aureus (ORSA) and enterococcus. Vancomycin was administered intravenously to the infant at the chronological age (CA) of 10 days. At the CA of 14 days, blood culture was positive for $C$. albicans. Amphotericin was administered intravenously until blood cultures were sterile. Threshold ROP with plus disease (Fig. 1) was diagnosed at gestational age (GA) of 36 weeks. The anterior segment of each eye also displayed a markedly dilated TVL and iris rigidity (Fig. 2). Avascular retinas in both eyes were treated with argon laser. Four weeks after retinal ablation, there was no evidence of retinal NV, plus disease (Fig. 3), TVL or iris rigidity (Fig. 4) in either eye. At 4-week follow-up, minor conjunctival swelling, and corneal edema were found, but there were no iris burn, posterior synechia, laser-induced retinal, or vitreous hemorrhage.

\section{DISCUSSION}

Extreme prematurity and ELBW are the most significant risk factors for candidemia in infants, as seen in our patient. Neonates with prolonged candidemia (positive blood culture for 5 days) are significantly more likely to develop ophthalmic, renal, or cardiac complications. ${ }^{1,2}$ However, early systemic antifungal therapy has been shown to decrease the frequency (from $45 \%$ to $6 \%$ ) of eye involvement in infants with candidemia. ${ }^{1,2,11,12}$ Candidemia in our patient did not lead to intraocular infections because the condition was treated early and aggressively with intravenous amophtericin B. Intralenticular infec-
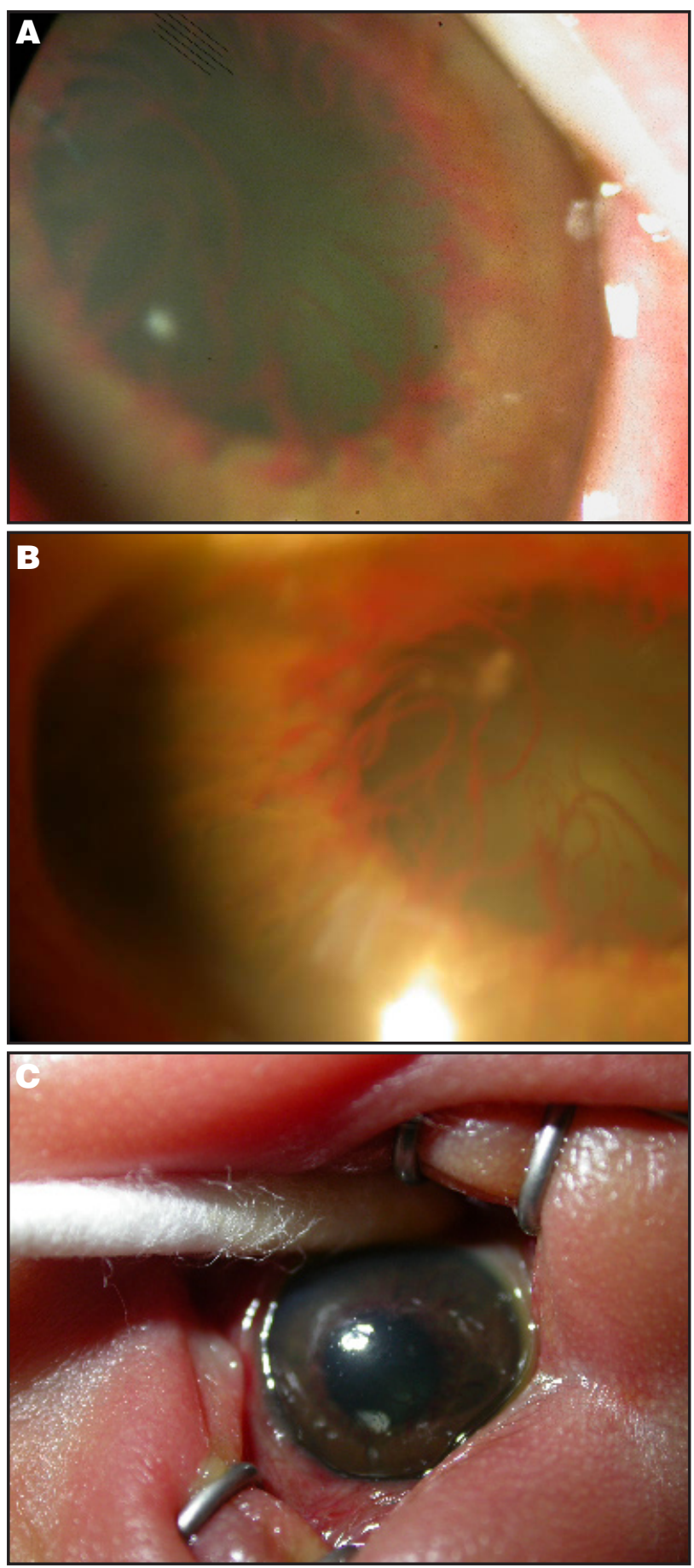

Figure 2. The anterior segment shows (A and $\mathbf{B})$ persistent TVL and $(\mathbf{C})$ iris rigidity.

tion can lead to cataract formation before regression of the TVL and the resulting infection nidus in a subsequently avascular structure of lens is poorly reached by antifungal drugs. Our patient did not develop lens abscess possibly because the systemic 

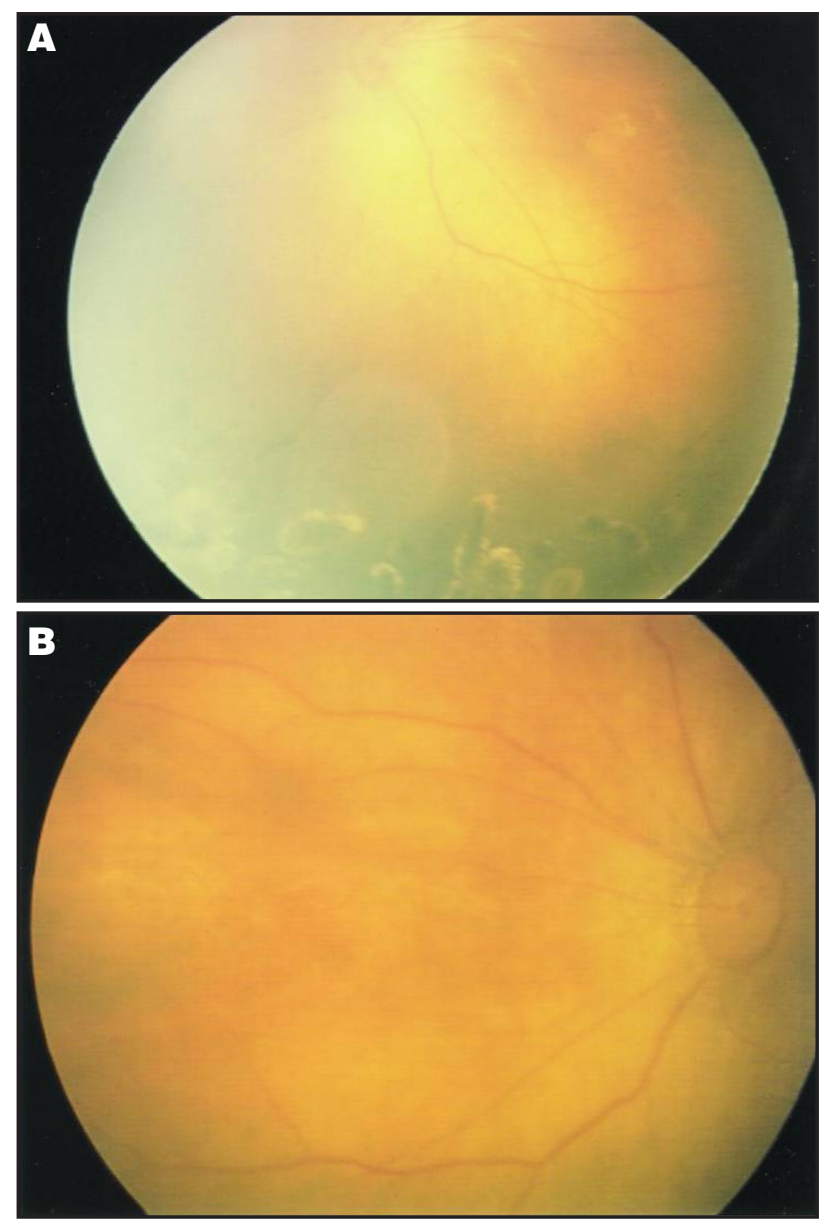

Figure 3. Extensive peripheral retinal neovascularization of the (A) left eye and (B) plus disease in the right eye completely regressed 4 weeks after laser therapy.

antifungal drug was administered before the lens became avascular.

Studies have shown that candidemia in preterm infants may progress to severe ROP requiring laser treatment. ${ }^{1,6-8}$ The reason for this progression is not known, but the authors suggest that candidemia may lead to over-expression of angiogenic substances, such as VEGF, thereby resulting in angiogenesis. ${ }^{1,6-8}$ C. albicans is known to interact with vascular endothelial cells in several ways. Candidemia may injure developing blood vessels in the retina, thereby increasing the chances for developing severe retinopathy. It is not known whether candidemia modulates the expression of known angiogenic factors (e.g. VEGF) which have been implicated in the pathogenesis of ROP. Further clinical or laboratory studies are needed to elucidate the exact mechanism by which candidemia exacerbates ROP.

TVL occurs rather frequently $(36.1 \%)$ in eyes with

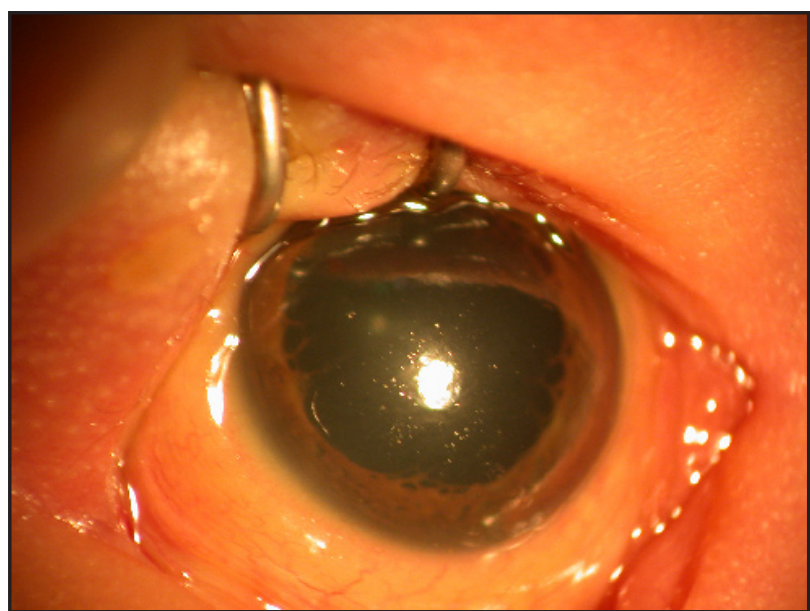

Figure 4. All signs of anterior segment involvement disappeared 4 weeks after laser therapy.

threshold ROP, ${ }^{13}$ as seen in our patient. VEGF, a hypoxia-induced cytokine, plays an important role in the normal development of retinal vasculature, in the involution of the HVS and in the development of ROP. ${ }^{4}$ In the proliferative phase of ROP, retinal ischemia induces increased levels of VEGF, which leads to retinal NV and persistent TVL. ${ }^{4}$ Clinically persistent TVL is often misdiagnosed as iris $\mathrm{NV},{ }^{10}$ a condition in which newly formed vessels develop during the iris surface from the pupil margin to the iris root. NV includes vasodilatation of the existing blood vessels, increased vascular permeability, degradation of the surrounding tissue, proliferation of endothelial cells, and formation of blood vessels. The TVL vessels run anterior, covering the lateral surface of the lens, and anastomose with the pupillary membrane, a vascular system supplied by the long posterior ciliary arteries. ${ }^{9}$ Except for the anatomically distinct vascular complex between iris NV and TVL, the TVL vessels differ from those of iris NV in possessing tight junctions between the endothelial cells that limit diffusion into the perivascular space. ${ }^{9}$ Iris NV and TVL vessels can be clinically differentiated by iris fluorescein angiography.

The signs of anterior segment, including persistent TVL, iris vessel engorgement, and iris rigidity are considered prognostic factors in ROP screening and in ROP treatment outcome. ${ }^{4,13}$ Elfein and Lorenz ${ }^{14}$ described twin sisters with TVL and severe ROP, and concluded that persistent and dilated TVL is a marker for acute severe ROP that may require treatment. Bagdoniene and Sirtautiene ${ }^{13}$ analyzed the importance of TVL in treatment outcome of threshold ROP and 
confirmed that eyes with persistent TVL and threshold ROP have a greater likelihood of unfavorable outcome after therapy; however, regression of TVL after therapy was shown to be directly related to favorable fundus outcomes. Seiberth et al. reported that diode laser photocoagulation can be used safely to treat acute ROP in patients with TVL. ${ }^{15}$ All unfavorable prognostic factors, including persistent TVL, iris vessel engorgement, and iris rigidity, were seen in our patient, but the threshold ROP with persistent TVL was successfully treated with argon laser therapy.

Although neonatal candidemia exacerbates the progression of ROP in an ELBW infant with persistent TVL, these conditions can be successfully treated with argon laser and early antifungal therapy. Ophthalmologists need to evaluate carefully with an indirect ophthalmoscope and follow-up closely for the progression of severe ROP and the development of eye infection, to render prompt and appropriate care.

\section{REFERENCES}

1. Baley JE, Ellis FJ. Neonatal candidiasis: ophthalmologic infection. Semin Perinatol. 2003;27:401-405.

2. Benjamin DK, Poole C, Steinbach W, Rowen JL, Walsh TJ. Neonatal candidemia and end-organ damage: a critical appraisal of the literature using meta-analytic techniques. Pediatrics. 2003;112:634-639.

3. The Committee for the Classification of Retinopathy of Prematurity. An international classification of retinopathy of prematurity. Arch Ophthalmol. 1984;102:1130-1134.
4. Travassos A, Teixeira S, Ferreira P, et al. Intravitreal Bevacizumab in aggressive posterior retinopathy of prematurity. Ophthalmic Surg Lasers Imaging. 2007;38:233-237.

5. Lutty GA, Chan-Ling T, Phelps DL, et al. Proceedings of the Third International Symposium on Retinopathy of Prematurity: An update on ROP from the lab to the nursery (November 2003, Anaheim, California). Mol Vis. 2006;12:532-580.

6. Mittal M, Dhanireddy R, Higgins RD. Candida sepsis and association with retinopathy of prematurity. Pediatrics. 1998;101:654-657.

7. Noyola DE, Bohra L, Paysse EA, Fernandez M, Coats DK. Association of candidemia and retinopathy of prematurity in very low birthweight infants. Ophthalmology. 2002;109:80-84.

8. Karlowicz MG, Giannone P, Pestian J, Morrow AL, Shults J. Does candidemia predict threshold retinopathy of prematurity in extremely low birth weight (1000 g) neonates? Pediatrics 2000;105:1036-1040.

9. Strek W, Strek P, Nowogrodaka-Zagorska M, Litwin JA, Pitynski K, Miodonski AJ. Hyaloid vessels of the human fetal eye: a scanning electron microscopic study of corrosion casts. Arch Ophthalmol. 1933; 111:1573-1577.

10. Prenner JL, Capone Jr A, Trese MT. Prominent tunica vasculosa lentis in a child with retinopathy of prematurity. Retina. 2003;23:267-268.

11. Drohan L, Colby CE, Brindle ME, Sanislo S, Ariagno RL. Candida (amphotericin-sensitive) lens abscess associated with decreasing arterial blood flow in a very low birth weight preterm infant. Pediatrics. 2002;110:1-4.

12. Shan GK, Vander J, Eagle RC. Intralenticular Candida species abscess in a premature infant. Am J Ophthalmol. 2000;129:390-391.

13. Bagdoniene R, Sirtautiene R. Clinical significance of tunica vasculosa lentis in the outcome of threshold ROP. Acta Medica Lituanica. 2006; 13:179-183.

14. Elfin HM, Lorenz B. Acute severe retinopathy of prematurely (ROP) in VLBW twin sisters: anterior and posterior segment findings as seen with serial digital wide field imaging. Invest Ophthalmol Vis Sci. 2005; 46:4130.

15. Rebirth V, Lindrtkamp O, Vardarli I, Knorz MC, Liesenhoff $\mathrm{H}$. Dioder laser photocoagulation for threshold retinopathy of prematurity in eyes with tunica vasculosa lentis. Am J Ophthalmol. 1995;119: 748-751. 